\title{
A MAPPING THEOREM FOR HILBERT CUBE MANIFOLDS
}

\author{
V. S. PRASAD ${ }^{1}$
}

\begin{abstract}
We show that every compact connected Hilbert cube manifold $M$ can be obtained from the Hilbert cube $Q$ by making identifications on a face of $Q$. Some applications of this result to measure preserving homeomorphisms on $M$ are given: (1) The first is concerned with which measures on $M$ are equivalent to each other by homeomorphisms. (2) The second application is about approximating invertible Borel measurable transformations of $M$ by measure preserving homeomorphisms of $M$. (3) The final application is concerned with generic properties of measure preserving homeomorphisms of $M$.
\end{abstract}

0. Introduction. Suppose $N$ is a compact connected $n$-manifold. Then it is easy to see that if $N$ is triangulable then $N$ can be obtained from the $n$-cube $\Delta^{n}$ by making identifications on the boundary $\partial \Delta^{n}$ (indeed, by knocking down adjacent walls in some triangulation of the manifold one obtains the result). In [B], M. Brown and B. Cassler proved that the same result is true even when the manifold is not triangulable.

In this note we obtain the analagous result for compact connected Hilbert cube manifolds. One proof of this result is merely the observation that the same proof that Brown and Cassler employ can be extended to cover the Hilbert cube manifold case. An alternate proof uses Chapman's result that every $Q$-manifold can be triangulated and then appeals to Brown's theorem in the triangulated case.

In $\S 2$, this theorem is used to show that many results concerning measure preserving homeomorphisms of Hilbert cubes can be extended to results about measure preserving homeomorphisms of Hilbert cube manifolds.

1. The mapping theorem. Let $Q=\Pi_{1}^{\infty}[0,1]$ be the Hilbert cube. By a $Q$-manifold $M$, we mean a separable metric manifold modeled on $Q$, with $d$ the metric on $M$.

THEOREM 1. Let $M$ be a compact connected Hilbert cube manifold. Then there is a continuous map $\Phi: Q \times[0,1] \rightarrow M$ such that:

(i) $\Phi$ is surjective.

(ii) $\Phi \mid Q \times[0,1)$ is a homeomorphism onto its image.

(iii) $\Phi(Q \times\{1\})$ is closed nowhere dense in $M$ and disjoint from $\Phi(Q \times[0,1))$.

Received by the editors February 8, 1982 and, in revised form, September 2, 1982. The contents of this paper were presented to the 793rd meeting of the American Mathematical Society, March 1982 at Bryn Mawr College.

1980 Mathematics Subject Classification. Primary 57N20; Secondary 28D05.

Key words and phrases. Hilbert cube manifold, measure preserving homeomorphism.

'Supported by grant no. U0080 from Natural Science and Engineering Research Council Canada. 
Proof. We offer two proofs.

The first proof is merely the observation that the simple proof given by Brown and Cassler works for the Hilbert cube manifold case if one engulfs by collared neighborhoods of the form $Q \times[0, r)$ in place of the balls of radius $r$; i.e. by using collared neighborhoods in $M$ of the form $Q \times[0, r)$ one can engulf most of the manifold (all except a closed nowhere dense subset of $M$ ). (Brown's proof can also be found in $[\mathbf{C}-\mathbf{V}]$.)

The second proof uses some deep results on $Q$-manifolds which can be found in [C]. I am indebted to both J. West and D. Curtis for suggesting this proof. Since every $Q$-manifold can be triangulated [C], $M$ is homeomorphic to $K \times Q$ for some compact connected polyhedron $K$. Let $N$ be a regular neighborhood of $K$ in some Euclidean space $R^{n}$. Since the natural retraction of $N$ onto $K$ is a simple homotopy equivalence, $N \times Q$ is homeomorphic to $K \times Q[\mathbf{C}]$. By Brown's theorem in the triangulated case, there exists a (piecewise linear) surjection $\phi: \Delta^{n} \rightarrow N$ such that $\phi \mid \check{\Delta}^{n}$ is a homeomorphism onto its image and $\phi^{-1} \phi\left(\partial \Delta^{n}\right)=\partial \Delta^{n}$. Let $h: I^{n} \times I \rightarrow \Delta^{n}$ $\times I$ be a homeomorphism such that $h\left(I^{n} \times[0,1)\right)=\Delta^{n} \times[0,1)$. Then define $\Phi$ : $Q \times[0,1] \rightarrow M$ as the composition

$$
I^{n} \times I \times Q_{n+1} \stackrel{h \times \mathrm{id}}{\rightarrow} \Delta^{n} \times I \times Q_{n+1} \stackrel{\phi \times \mathrm{id} \times \mathrm{id}}{\rightarrow} N \times I \times Q_{n+1} \stackrel{g}{\rightarrow} M
$$

where $g$ is any homeomorphism and $Q=\Pi_{1}^{\infty}[0,1]=I^{n} \times Q_{n+1}$.

2. Measure preserving homeomorphisms. Theorem 1 may be of interest to $Q$-manifold topologists; however our interest in this result was motivated by its applications to the study of measure preserving homeomorphisms of $M$.

Let $\mu$ be a nonatomic normalised Borel measure on $M$ which is locally positive (i.e. positive on open sets). Let $\mathcal{H}(M)$ be the set of homeomorphisms of $M$ onto itself, and $\mathcal{H}_{\mu}(M)$ the subset of $\mu$-preserving homeomorphisms of $M$. The metric $\bar{d}(h, g)$ $=\sup _{x \in M} d(h(x), g(x))+\sup _{x \in M} d\left(h^{-1}(x), g^{-1}(x)\right)$ for $h, g \in \mathcal{H}(M)$ makes $\mathcal{H}(M)$ a complete metric space (and $\mathcal{H}_{\mu}(M)$ is a closed subset of $\mathcal{H}(M)$ ). For $A \subset M$ let $\mathcal{H}(M, A)=\{h \in \mathcal{H}(M): h$ is the identity on $A\}$.

Proposition. Let $M$ be a compact connected Hilbert cube manifold and let $\nu$ be any $\sigma$-finite Borel measure on $M$. If $F$ is a closed nowhere dense subset of $M$, then $\{h \in \mathcal{H}(M): \nu h(F)=0\}$ is a dense $\mathcal{G}_{\delta}$ set in $\mathcal{H}(M)$.

Proof. Let $D_{1}, \ldots, D_{k}$ be a covering of $M$ by sets homeomorphic to $Q$ and such that $\nu\left(\partial D_{i}\right)=0, i=1, \ldots, k$. If $i \in\{1, \ldots, k\}$ and $j \in \mathbf{N}$ define $\xi(i, j)=\{h \in$ $\left.\mathcal{H}(M): \nu\left(h F \cap D_{i}\right)<1 / j\right\}$. Then $\{h \in \mathcal{H}(M): \nu h(F)=0\}=\bigcap_{i, j} \xi(i, j)$. We need to show that $\xi(i, j)$ is open and dense.

Given $g \in \mathcal{H}(M)$, the set $g F \cap D_{i}$ is closed nowhere dense in $D_{i}$. By Theorem 7 [O-P] with $\partial D_{i}$ a $Z$-set in $D_{i}$, we can find an arbitrarily small homeomorphism $f \in \mathcal{H}\left(D_{i} ; \partial D_{i}\right)$ such that $\nu\left(f g F \cap D_{i}\right)<1 / j$. Extend $f$ to $M$ by setting $f$ equal to the identity outside $D_{i}$. Then $f g \in \xi(i, j)$ and, since $f$ is small, $f g$ is close to $g$, proving $\xi(i, j)$ is dense. 
The subset $\xi(i, j)$ is open: let $h_{0} \in \xi(i, j)$. Then we can find an open set $U \supset h_{0}(F) \cap D_{i}$ so that $\nu(U)<1 / j$. Choose $h \in \mathcal{H}(M)$ sufficiently close to $h_{0}$ so that $h(F) \cap D_{i} \subset U$ also. Then $h \in \xi(i, j)$. This completes the proof of the Proposition.

Hence we can assume in Theorem 1 that $\nu(\Phi(Q \times\{1\}))=0$. Thus, $M$ contains a set $M^{\prime}$ homeomorphic to $Q \times[0,1)$ with $\nu\left(M \backslash M^{\prime}\right)=0$. We now apply this refinement of Theorem 1 to obtain the following extension to $M$ of the homeomorphic measures theorem for $Q$ (cf. [O-P, Theorem 1]).

THEOREM 2. Let $\mu$ and $\nu$ be two nonatomic, normalised, locally positive Borel measures on $M$, a compact connected Hilbert cube manifold. Then there is $h \in \mathcal{H}(M)$ such that $\mu(E)=\nu h(E)$ for all Borel sets $E \subset M$.

Proof. By applying the refinement of Theorem 1 to the sum measure $\mu+\nu$, we may assume that $\mu(\Phi(Q \times\{1\}))=\nu(\Phi(Q \times\{1\}))=0$. We define measures $\mu^{\prime}$ and $\nu^{\prime}$ on $Q \times[0,1]$ by setting $\mu^{\prime}(E)=\mu \Phi(E)$ and $\nu^{\prime}(E)=\nu \Phi(E)$ for each Borel set $E \subset Q \times[0,1]$. Then $\mu^{\prime}, \nu^{\prime}$ are nonatomic, normalised, locally positive Borel measures on $Q \times[0,1]$ which are zero on the face $B=Q \times\{1\}$. Hence, by [O-P, Theorem 1], there is $h^{\prime} \in \mathcal{H}(Q \times[0,1] ; B)$ such that $\mu^{\prime}=\nu^{\prime} h^{\prime}$. Then the map $h=\Phi h^{\prime} \Phi^{-1}$ is a homeomorphism of $M$ which fixes $\Phi(Q \times\{1\})$, and for which $\mu=\nu h$.

We can also use Theorem 1 to obtain a measure preserving Luzin type theorem for $M$ (cf. [O-P, Theorem 5] for the Hilbert cube case).

THEOREM 3. Let $\mu$ be a nonatomic, normalised, locally positive Borel measure in $M$. Let $T$ be an invertible Borel measurable and $\mu$-preserving transformation of $M$ onto itself. For each $\varepsilon>0$ there exists a $\mu$-preserving homeomorphism $h \in \mathcal{H}(M)$ such that $\mu(\{x \in M: h x \neq T x\})<\varepsilon$.

Proof. Assuming that $\mu(\Phi(Q \times\{1\}))=0$ define the measure $\mu^{\prime}$ in $Q \times[0,1]$ by setting $\mu^{\prime}(E)=\mu(\Phi(E))$ for each Borel set $E \subset Q \times[0,1]$. Then $\mu^{\prime}$ is a nonatomic normalised locally positive Borel measure in $Q$ which is zero on the face $B=Q \times\{1\}$.

Define

$$
\tilde{T} x= \begin{cases}x & \text { if } x \in \cup_{n \in \mathbf{z}} T^{n} \Phi(B), \\ T x & \text { otherwise. }\end{cases}
$$

Then $\tilde{T} x=T x \mu$-a.e. $x \in M$ and $\tilde{T} x=x$ for $x \in \Phi(B)$ and $T^{\prime}=\Phi^{-1} \tilde{T} \Phi$ defines an invertible $\mu^{\prime}$-preserving Borel measurable transformation of $Q \times[0,1]$ onto itself. Hence by [O-P, Theorem 5], there is $h^{\prime} \in \mathcal{H}(Q \times[0,1] ; B)$ a $\mu^{\prime}$ preserving homeomorphism of $Q \times[0,1]$ onto itself such that $\mu^{\prime}\left\{x \in Q \times[0,1]: h^{\prime} x \neq T^{\prime} x\right\}<\varepsilon$. Setting $h=\Phi h^{\prime} \Phi^{-1}$ gives us the required $\mu$-preserving homeomorphism $h$ of $M$ so that $\mu\{x \in M: h x \neq T x\}<\varepsilon$.

Theorem 1 can also be used to extend the results of Alpern $\left[\mathbf{A}_{2}\right]$ to study generic properties of measure preserving homeomorphisms of a compact connected Hilbert cube manifold. Indeed such an extension was anticipated in $\left[\mathbf{A}_{2}\right]$ but requires our Theorem 1. 
For $\mu$ a nonatomic, normalised locally positive Borel measure on $M$, let $\Re(M)$ be the set of invertible $\mu$-preserving Borel measurable transformations of $M$ onto itself. On $\Re(M)$ we put the Halmos weak topology (see $\left[\mathbf{A}_{1}\right]$ or $\left[\mathbf{A}_{2}\right]$ ).

THEOREM 4. Let $\bigodot \subset \mathfrak{N}(M)$ be any conjugate invariant subset of $\mathfrak{N}(M)$ which is a dense $\mathcal{G}_{\delta}$ set in $\mathfrak{T}(M)$ with the weak topology. Then $\mathcal{\cap} \mathcal{H}_{\mu}(M)$ is a dense $\mathcal{G}_{\delta}$ in $\mathcal{H}_{\mu}(M)$ with the topology of uniform convergence.

Proof. In [ $\mathbf{A}_{2}$, p. 26], Alpern proves the result for the Hilbert cube $Q$ and any locally positive, nonatomic, normalised Borel measure. He also shows that the same proof applies when the space $(M, d, \mu)$ is obtained from the Hilbert cube by making identifications on a face.

Hence, since as a measure space $(M, \mu)$ is just a Lebesgue space, we can thus assert for example, the existence of $\mu$-preserving homeomorphisms of $M$ which are ergodic or weak mixing.

\section{REFERENCES}

[A $\left.\mathbf{A}_{1}\right]$ S. Alpern, Approximation to and by measure preserving homeomorphisms, J. London Math. Soc. (2) 18 (1978), 305-315.

$\left[\mathbf{A}_{2}\right]$, Generic properties of measure preserving homeomorphisms, Ergodic Theory (Proc., Oberwolfach, Germany, 1978), Lecture Notes in Math., vol. 729, Springer-Verlag, Berlin and New York, 1979, pp. 16-27.

[B] M. Brown, A mapping theorem for untriangulated manifolds, Topology of 3-Manifolds and Related Topics (M. K. Fort, editor), Prentice-Hall, Englewood Cliffs, N. J., 1963, pp. 92-94.

[C] T. A. Chapman, Lectures on Hilbert cube manifolds, CBMS Regional Conf. Ser. in Math., no. 28, Amer. Math. Soc., Providence, R. I., 1976.

[C-V] C. Christenson and W. Voxman, Aspects of topology, Dekker, New York, 1977.

[O-P] J. C. Oxtoby and V. S. Prasad, Homeomorphic measures in the Hilbert cube, Pacific J. Math. 77 (1978), 483-497.

Departement de Mathematiques, Universite de Sherbrooke, Sherbrooke, Quebec, Canada J1K 2R 1 